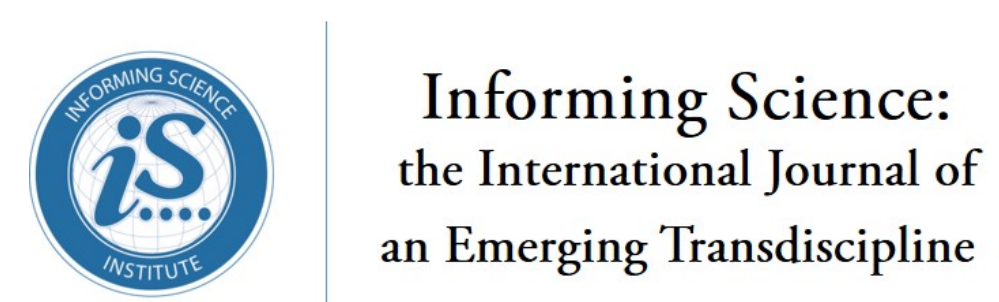

An Official Publication

of the Informing Science Institute

InformingScience.org

Inform.nu

Volume 21, 2018

\title{
A SOCIAL MACHINE FOR TRANSDISCIPLINARY RESEARCH
}

David G. Lebow

HyLighter LLC, Tallahassee, FL USA

david@hylighter.com

\section{ABSTRACT}

Aim/Purpose

Background

Methodology

Contribution

Findings

Recommendations for Practitioners

Recommendations for Researchers

Impact on Society
This paper introduces a Social Machine for collaborative sensemaking that the developers have configured to the requirements and challenges of transdisciplinary literature reviews.

Social Machines represent a promising model for unifying machines and social processes for a wide range of purposes. A development team led by the author is creating a Social Machine for activities that require users to combine pieces of information from multiple online sources and file types for various purposes.

The development team has applied emergent design processes, usability testing, and formative evaluation in the execution of the product road map.

A major challenge of the digital information age is how to tap into large volumes of online information and the collective intelligence of diverse groups to generate new knowledge, solve difficult problems, and drive innovation. A Transdisciplinary Social Machine (TDSM) enables new forms of interactions between humans, machines, and online content that have the potential to (a) improve outcomes of sensemaking activities that involve large collections of online documents and diverse groups and (b) make machines more capable of assisting humans in their sensemaking efforts.

Preliminary findings suggest that TDSM promotes learning and the generation of new knowledge.

TDSM has the potential to improve outcomes of literature reviews and similar activities that require distilling information from diverse online sources.

TDSM is an instrument for investigating sensemaking, an environment for studying various forms of human and machine interactions, and a subject for further evaluation.

In complex areas such as sustainability and healthcare research, TDSM has the potential to make decision-making more transparent and evidence-based, facilitate the production of new knowledge, and promote innovation. In education, TDSM has the potential to prepare students for the $21^{\text {st }}$ century information economy.

This paper is one in a Special Series on Transdisciplinary Communication

Accepting Editor Shalini Misra | Received: November 13, 2017 | Revised: March 24, April 12, 2018 | Accepted: April 16, 2018.

Cite as: Lebow, D. G. (2018). A social machine for transdisciplinary research. Informing Science: the International Journal of an Emerging Transdiscipline, 21, 201-217. https://doi.org/10.28945/4025

(CC BY-NC 4.0) This article is licensed to you under a Creative Commons Attribution-NonCommercial 4.0 International License. When you copy and redistribute this paper in full or in part, you need to provide proper attribution to it to ensure that others can later locate this work (and to ensure that others do not accuse you of plagiarism). You may (and we encourage you to) adapt, remix, transform, and build upon the material for any non-commercial purposes. This license does not permit you to use this material for commercial purposes. 
Future Research Research is required to measure the effects of TDSM on cross-disciplinary communication, human and machine learning, and the outcomes of transdisciplinary research projects. The developers are planning a multiple case study using designbased research methodology to investigate these topics.

Keywords cognitive bias, epistemological beliefs, multiple texts, sensemaking, Social Machines, transdisciplinarity, transdisciplinary research

\section{INTRODUCTION}

This paper introduces a Social Machine for transdisciplinary literature reviews (i.e., the exploration of various topics and publications through multiple disciplinary and theoretical lenses; Montuori, 2013) and for similar activities that require an individual or group to combine pieces of information from multiple sources for various purposes. Berners-Lee and Fischetti (1999) defined a Social Machine as a socio-technical construct where machines help humans to enhance creativity, facilitate collaboration, and increase global connectivity. At the time, they predicted that Social Machines would be central to the evolution of the Web and future relationships between humans and machines. Over the past two decades, Social Machines have become the focus of large-scale research programs (e.g., the SOCIAM initiative from EPSRC, the Laboratory for Social Machines at MIT) and many academic publications (Smart \& Shadbolt, 2014). A common theme across diverse perspectives is that Social Machines represent a promising model for unifying machines and social processes (i.e., interactions between individuals and groups that influence attitudes, behaviors, and outcomes) for a wide range of purposes (Buregio, Meira, \& Rosa, 2013).

Today, Social Machines are enabling new forms of social interaction and coordination through a variety of Web-based applications. These include, among others, Wikipedia—a collaborative editing site for democratically producing an encyclopedia, Facebook — a social networking system for allowing people to interact with other users, GalaxyZoo—a crowdsourcing approach for distributing the massive task of classifying galaxies and stars, and OpenRov—a distributed sensor network composed of underwater drones and their human handlers for exploring the ocean (Smart, Simperl, \& Shadbolt, 2014). A Social Machine for supporting transdisciplinarity may help improve outcomes in such critical areas as sustainability and healthcare research.

As Knorr-Cetina (1999) wrote, science is not a unitary enterprise, but a market of independent epistemic monopolies producing vastly different products through different epistemic machinery (i.e., "methodologies, techniques, tools and instruments used in our knowledge production and distribution;" Mørk, Aanestad, Hanseth, \& Grisot, 2008, p.15). Extending this metaphor, integrative research (i.e., multi-, inter- and transdisciplinary) holds the promise of innovative products and solutions for teams of diverse members who are willing to relinquish their disciplinary monopolies.

Over the past fifty years, theorists have proposed various descriptions and definitions for distinguishing different approaches to integrative research (J. T. Klein, 2008a). The terms multidisciplinary, interdisciplinary, and transdisciplinary have frequently appeared in the literature but with varying and inconsistent definitions. For current purposes, multidisciplinarity takes into account knowledge from different disciplines but involves little interaction across boundaries. Interdisciplinarity involves interaction across boundaries with potential to affect the perspectives and research outputs of disciplinary members. Transdisciplinarity involves a creative synthesis where members of different disciplines transcend boundaries to form a new, integrated, and more holistic approach (Darbellay, 2014; J. T. Klein, 2008b; Rosenfield, 1992; Stock \& Burton, 2011). In other words, multidisciplinary is additive, interdisciplinary is interactive, and transdisciplinary is holistic (Choi \&Pak, 2006).

These distinctions aside, all approaches to integrated research face various barriers (e.g., institutional, compositional, contextual, and emotional) to productive cross-disciplinary collaboration (Salazar, Lant, Fiore, \& Salas, 2012). Transdisciplinary research faces the additional challenge of reconciling 
differences in epistemic cultures (i.e., "cultures that create and warrant knowledge;" Knorr-Cetina, 1999, p.1) to generate new and integrated knowledge. Succinctly stated, the main qualities of transdisciplinarity include the following: (a) focus on socially relevant issues, (b) aim for a creative synthesis of disciplinary perspectives, (c) make research participatory and inclusive, and (d) pursue a unity of knowledge (Pohl, 2010). Consistent with this perspective, transdisciplinarity asserts that no one discipline sits in a privileged position for meeting the many problems and threats facing humanity (Vilsmaier, Brandner, \& Engbers, 2017).

A development team led by the author has created a Transdisciplinary Social Machine (TDSM) to help address the challenges facing transdisciplinary research teams. TDSM enables machines as specially-abled teammates to assist humans in addressing three key obstacles commonly encountered in performing a transdisciplinary literature review. These include (a) overcoming differences in language and meaning between disciplines, (b) coping with information overload in attempting to synthesize information from multiple sources and disciplines, and (c) avoiding potentially negative effects of cognitive bias.

The following narrative about TDSM has four main headings:

1. Three Problems in Performing a Transdisciplinary Literature Review: identifies three challenges facing a transdisciplinary research team performing a literature review that TDSM addresses.

2. Social Machine Components and Framework: describes the main components of TDSM.

3. Three Examples of Machine-Assisted Sensemaking: describes how a team uses the system to address identified problems in performing a literature review.

4. Conclusion: summarizes the paper and reflects on the potential of TDSM to help prepare students and others for the information economy and an uncertain future.

\section{Three Problems in Performing a Transdisciplinary LITERATURE REVIEW}

In a world facing numerous, intractable problems that refuse to fit neatly into disciplinary boxes, transdisciplinarity takes a holistic approach. However, teams of diverse stakeholders, including scientists from multiple disciplines and non-scientists, face major stumbling blocks in their attempts to collaborate across epistemic boundaries. Keys to success include the ability of a team to develop integrative capacity and team effectiveness through quality of communication, ability to acquire and marshal relevant information, and attention to group process. Integrative capacity is an emergent property of social and cognitive processes that shape the ability of a team to overcome obstacles to effective collaboration and combine knowledge from diverse perspectives (Salazar et al., 2012). Team effectiveness is the capacity of a group of individuals to accomplish the goals of individual members and the goals shared by the group (Mathieu, Maynard, Rapp, \& Gibson, 2008). From a related perspective, readiness for collaboration may influence outcomes for transdisciplinary research projects as determined by level of institutional support, prior experience of members with collaborative projects, and degree of epistemological and ontological incompatibilities, among other considerations (Hall et al., 2008).

To illustrate the potential value of Social Machines for performing transdisciplinary literature reviews, we focus on three particularly challenging problems. These include (a) differences in language and meaning, (b) information overload, and (c) effects of cognitive bias.

\section{DIFFERENCES IN LANGUAGE AND MEANING}

Differences in terminology is a major barrier to transdisciplinarity, especially for teams with high diversity (Hall \& Vogel, 2012). Practitioners within different disciplines use specialized terms or jargon, use different terms to talk about the same object, and use the same terms but attach different mean- 
ings. Teams that fail to develop a shared language are limited in their capacity to establish a shared mental model (i.e., an organized representation of knowledge or negotiated belief structure that is shared by team members; Mathieu et al., 2008; Walsh \& Fahey, 1986) as a basis for participating in the integration of ideas from different disciplines.

To further complicate matters, transdisciplinary research inherently involves two simultaneous projects. One is to collaboratively define and address a problem, and the other is to reconfigure disciplinary boundaries to establish a new hybrid or "culture of emergence" (Ciliers, 1998; Robinson, 2008). From another perspective, establishing a new hybrid means fabricating new epistemic machinery to meet the requirements of the current context (i.e., by selectively integrating technology, methods, and ideas appropriated from different disciplines). To accomplish these interrelated projects, a team must develop a shared language for integrating multiple perspectives on the defined problem and defining what transdisciplinarity means within the current context.

\section{INFORMATION OVERLOAD}

Today, people are inundated with so much information, located in so many different places, that they frequently have trouble finding essential information and recognizing important associations between related pieces of information (Misra, Hall, Feng, Stipleman, \& Stokols, 2011). The problem is compounded when organizational silos cut people off from expertise and information and projects require collaboration among people with diverse perspectives.

Transdisciplinary research teams commonly undertake literature reviews that cross multiple disciplinary boundaries. Considering that approximately 2.5 million research papers are published in over 28,100 journals annually (Boon, 2016), occupational hazards facing team members include feeling overwhelmed by the volume of available content scattered in different fields and worrying about what key ideas might be missing from the picture (i.e., unknown unknowns).

Adding further to the challenges of transdisciplinarity, when researchers read literature outside of their own fields, they are at risk of having insufficient breadth of knowledge to know which pieces of information are relevant. Also, they are less likely to recognize non-obvious and meaningful associations across disciplinary literature - a key source of serendipitous discovery and innovation (Salazar et al., 2012).

\section{NEGATIVE EFFECTS OF COGNITIVE BIAS}

Literature reviews frequently involve reconciling and resolving alternative views on the same or related issues. In transdisciplinary literature reviews, team members face the added challenge of achieving a creative synthesis of diverse views through interdisciplinary integration. This not only requires a high-level of coordination and trust but, also, a large measure of open-mindedness (i.e., receptiveness to new ideas, including ideas that conflict with current beliefs) among individuals who tend to see the world through different lenses (Shattuck \& Miller, 2006).

According to Richter and Maier (2017), epistemic monitoring (i.e., a routine and continuously operating process in the evaluation of incoming information for internal consistency and plausibility) serves as a filtering device in reading comprehension. Information consistent with current beliefs acquires a processing advantage over inconsistent information in comprehension and memory. In other words, prior knowledge and beliefs of readers serve a gatekeeper function in processing information. In general, readers evaluate information in texts that is consistent with their beliefs as more plausible than information that is inconsistent with their beliefs (Richter, 2011). They also tend to value evidence that supports what they are motivated to believe and discredit evidence that contradicts what they believe (Golman, Hagmann, \& Loewenstein, 2017).

Beliefs about the nature of knowledge and knowing, referred to as epistemological beliefs, among other terms (e.g., epistemic cognition (Greene, Azevedo, \& Torney-Purta, 2008); personal epistemol- 
ogy (Hofer, 2004); and epistemic beliefs; Schraw, Bendixen, \& Dunkle, 2002), influence cognitive flexibility (i.e., ability and willingness to adopt some arguments and reject others on rational grounds; Misra, Stokols, \& Cheng, 2015; Stokols, 2014). Epistemological beliefs are relatively stable characteristics that appear to affect individual engagement in epistemic elaboration (i.e., effortful activity to critically evaluate information that conflicts with current beliefs; Richter, 2011). In the context of a literature review, epistemological beliefs of individual team members may inhibit cognitive flexibility.

Research on epistemic beliefs and epistemic monitoring is consistent with a growing body of literature in both psychology (e.g., Golman et al., 2017) and economics (e.g., Babcock, Loewenstein, Issacharoff, \& Camerer, 1995) on the vulnerability of humans to various forms of cognitive bias (i.e., an umbrella term covering a diverse typology of systematic errors in judgment and decision-making that are prevalent in all human beings; Kahneman, Slovic, \& Tversky, 1992). Table 1 describes several forms of cognitive bias that researchers have identified and investigated.

Table 1. Examples of Cognitive Bias

\begin{tabular}{|l|l|}
\hline Cognitive inflexibility & $\begin{array}{l}\text { Individuals fail to flexibly adapt their thinking and actions to } \\
\text { deal with changes in the environment. For example, (a) an in- } \\
\text { dividual insistently takes actions that were effective in a previ- } \\
\text { ous situation while in a new situation where they are ineffective } \\
\text { and (b) an expert relies too heavily on automated performance } \\
\text { routines and, as a result, is less able to judge the likelihood of a } \\
\text { failure in a system (Canas, Fajardo, \& Salmeron, 2006). }\end{array}$ \\
\hline Text-belief consistency effect & $\begin{array}{l}\text { Readers evaluate information in texts that is consistent with } \\
\text { their beliefs as more plausible than information that is incon- } \\
\text { sistent with their beliefs (Richter \& Maier, 2017). }\end{array}$ \\
\hline Active and passive information & $\begin{array}{l}\text { People use diverse tactics to avoid obtaining information, ei- } \\
\text { ther intentionally or unintentionally (Golman et al., 2017). }\end{array}$ \\
\hline Confirmation bias & $\begin{array}{l}\text { People have a tendency to interpret new information in a way } \\
\text { that confirms their preexisting beliefs or theories (Rabin \& } \\
\text { Schrag, 1999). }\end{array}$ \\
\hline Bounded awareness & $\begin{array}{l}\text { Individuals regularly fail to see and use information easily } \\
\text { available to them, and groups ignore unique or unshared in- } \\
\text { formation held by group members while focusing on shared } \\
\text { information (Chugh \& Bazerman, 2007). }\end{array}$ \\
\hline Focalism or focusing failure & $\begin{array}{l}\text { People over-focus on selected information and ignore other } \\
\text { easily available and pertinent information, resulting in ill- } \\
\text { informed decisions (Chugh \& Bazerman, 2007). }\end{array}$ \\
\hline Ingroup favoritism & $\begin{array}{l}\text { Individuals select material for carrying out a literature review } \\
\text { that is biased toward reaching specific conclusions (Lavallée, } \\
\text { Robillard, \& Mirsalari, 2014). }\end{array}$ \\
\hline Completeness bias & $\begin{array}{l}\text { Group members demonstrate limited willingness to share } \\
\text { knowledge with or value the contributions from individuals } \\
\text { outside of their own discipline or community of practice (La- } \\
\text { vallée et al., 2014). }\end{array}$ \\
\hline $\begin{array}{l}\text { Individuals adopt the prevailing opinion of a group as their } \\
\text { own, at the expense of creativity and individual responsibility } \\
\text { (Golman et al., 2017). }\end{array}$ \\
\hline
\end{tabular}


According to Schacter (2001), biases are not design flaws in human memory and thinking but are byproducts of adaptive features. In other words, benefits inevitably come with trade-offs and disadvantages. For example, cognitive biases, viewed as information-processing shortcuts, may help the brain to quickly make sense of information and experiences (Fay \& Montague, 2014). On the other hand, they may lead to systematic errors in judgment and decision-making (Kahneman et al., 1992).

This notion seems consistent with the data-frame theory of sensemaking. As defined by G. Klein, Phillips, Rall, and Peluso (2007), a frame is "an explanatory structure that defines entities by describing their relationships to other entities" (p.118). (According to the authors, rather than trying to reconcile distinctions between frames, scripts, schemata, and mental models, they chose to use the term frame as a synthesis of these concepts.) As data accumulates, the sensemaker will make decisions about whether to incorporate new information into the current frame or generate a new frame that better accounts for the new information. Consistent with Rumelhart and Norman (1978), updating an existing frame (i.e., accretion and tuning) requires less time and mental effort than re-framing or generating a new frame (i.e., restructuring).

Given that members of a discipline tend to narrow their focus to information within their own discipline, they may ignore information that does not fit within their disciplinary boundaries (Montuori, 2013). From this perspective, disciplinary literacy (i.e., approaches to reading, writing, thinking, and reasoning shared by members within academic fields; Shanahan \& Shanahan, 2008) may inhibit cognitive flexibility (Mulane \& Williams, 2014; Smith \& Noble, 2014). In a sense, each discipline trains and enculturates its initiates in practices of sanctioned cognitive biases.

While many fields of knowledge are becoming increasing specialized, partly in response to the information tsunami of the digital age, transdisciplinarity points in a different direction. What sets transdisciplinarity apart from unidisciplinarity and other forms of integrative research (e.g., multiand inter-disciplinarity) is its holistic perspective on the nature of knowledge and knowing. At its core, transdisciplinarity is about the creative synthesis of knowledge to address real-world problems through transcending ideological, scientific, religious, economic, political and philosophical boundaries (Shrivastava \& Ivanaj, 2011). Ultimately, successful implementation requires teams to establish conditions that diminish disciplinary chauvinism and cognitive biases and favor transparency, selfreflection and willingness to question personal assumptions and beliefs (Stock \& Burton, 2011).

\section{SOCIAL MACHINE COMPONENTS AND FEATURES}

To assist teams in implementing a transdisciplinary literature review, we have designed and partially implemented a Transdisciplinary Social Machine (TDSM). The general function of the system is to support users in accomplishing activities that require individuals or groups to combine pieces of information from multiple online sources and file types for various purposes. The main theoretical framework underlying the design of the system draws on the learning sciences and, most centrally, on the concept of sensemaking.

Theorists have offered a variety of definitions for sensemaking (also, sense-making and sense making) and descriptions of sensemaking behaviors (e.g., G. Klein, Moon, \& Hoffman, 2006; Pirolli \& Card, 2005; Slaney \& Russell, 2005; Weick, Sutcliffe, \& Obstfeld, 2005). Within TDSM and the context of performing a transdisciplinary literature review, we define sensemaking as the process of finding, organizing, and synthesizing high-value information from multiple documents and the collective intelligence of diverse group members to generate new knowledge and achieve other desirable outcomes.

TDSM contains four main sensemaking components including (a) the graphical user interface (GUI), (b) the communication or message bus, (c) the breadboard or plugboard, and (d) machine component plugins. Table 2 describes these core sensemaking components. 
Table 2. Main Components and Features for a Transdisciplinary Social Machine

\begin{tabular}{|c|c|}
\hline COMPONENTS & FEATURES \\
\hline $\begin{array}{l}\text { 1. TDSM Graphical User Interface } \\
\text { (GUI) } \\
\text { A Web-based environment for } \\
\text { collaborative sensemaking activ- } \\
\text { ities that requires users to com- } \\
\text { bine pieces of information } \\
\text { from multiple online sources } \\
\text { and file types for various pur- } \\
\text { poses. }\end{array}$ & $\begin{array}{l}\text { O Provides a graphical user interface for managing and } \\
\text { participating in sessions, mashups, and Knowledge } \\
\text { Trails. } \\
\text { - Session: an individual document that includes hu- } \\
\text { man and/or machine generated HyLights. } \\
\text { - HyLight: a highlighted fragment of text or an area } \\
\text { of an image and related meta-content or content } \\
\text { about content (e.g., author ID, comments, } \\
\text { date/time stamps, tags, and links). } \\
\text { - Mashup: a set of HyLights excerpted from multi- } \\
\text { ple sessions with links back to their exact locations } \\
\text { within their sources. } \\
\text { Knowledge Trail: a set of HyLights that a user has } \\
\text { selected from multiple documents (i.e., a type of } \\
\text { mashup), arranged in a preferred order (e.g., a nar- } \\
\text { rative structure) and saved to a library for reuse } \\
\text { and sharing. }\end{array}$ \\
\hline $\begin{array}{l}\text { 2. Communication Bus } \\
\text { A mechanism for efficiently } \\
\text { managing streams of events } \\
\text { within the system. }\end{array}$ & $\begin{array}{l}\text { Provides the system with a medium for internal and } \\
\text { external components to publish and subscribe to mes- } \\
\text { sages about activities in the system. }\end{array}$ \\
\hline $\begin{array}{l}\text { 3. Breadboard } \\
\text { A mechanism for wiring ma- } \\
\text { chine components together. }\end{array}$ & $\begin{array}{l}\text { Provides a wiring mechanism for establishing explicit } \\
\text { connections between machine components that allow } \\
\text { for complex interactions among components. }\end{array}$ \\
\hline $\begin{array}{l}\text { 4. Machine Component Plugins } \\
\text { A mechanism that adapts ma- } \\
\text { chine behaviors for use within } \\
\text { TDSM. }\end{array}$ & $\begin{array}{l}\text { Provides a set of interface definitions that allow inter- } \\
\text { nal or external components to participate in TDSM } \\
\text { through software adapters. }\end{array}$ \\
\hline
\end{tabular}

\section{THE TDSM SENSEMAKING GUI}

The design of the TDSM graphical user interface (GUI) is informed by user-experience design principles with the goal of enabling machines to assist non-technical people (and data scientists, too) in sensemaking activities. (An example of how a machine might participate in this process is described later below.) The GUI has provisions for importing a wide variety of document file types (e.g., PDFs, Office files, and most common image files). Upon import, the system creates high-fidelity copies of the original files as HTML. The converted files display in most browsers and allow for annotating user-selected fragments of text or areas of images.

The TDSM GUI enables users to add HyLights to documents, manage access and permissions to their sessions, and create mashups of HyLights from multiple documents. A HyLight is an annotation that includes a color-coded fragment and related meta-content (i.e., content about content) such as author ID, comments, date/time stamps, tags, and a specific URL for the HyLight. A session is an individual document that displays HyLights accessible to a given user for the selected document. A mashup is a set of HyLights excerpted from multiple documents with links back to their exact locations within their sources (e.g., results of a search or user selections). TDSM stores HyLight information in a relational database. As requested, the system overlays color-coded highlighting on select- 
ed pages, displays HyLight information from the current document in the left session-margin, and displays HyLight information from multiple documents in the right mashup-margin.

In addition to creating HyLights, users can (a) reply to an existing HyLight, (b) establish a link between two HyLights in different documents, (c) search and filter on HyLights and full document text, (d) add HyLights from a session to a mashup, and (e) create Knowledge Trails. A Knowledge Trail is a set of HyLights that a user (i.e., an individual, a group, a machine, or a combination of people and machines) has filtered or distilled from multiple documents (i.e., a type of mashup), arranged in a preferred order (e.g., a narrative structure), and saved to a library for reuse and sharing. To create a Knowledge Trail, a user (a) arranges selected HyLights displayed in the right mashup-margin into a preferred sequence (e.g., using cut \& paste, among other features), (b) names and saves the results to a library for revisiting, repurposing, and sharing with others, and, optionally, (c) exports or publishes the contents to an external application or file type (e.g., Word, Excel, Acrobat, or GoogleDocs). A key attribute of mashups, including Knowledge Trails, is that they provide nearly instantaneous navigation between HyLights. This capability enables rapid review of HyLights located in different documents, which may accelerate learning and increase serendipity (e.g., by helping users to make nonobvious associations between ideas that are distant from each other).

\section{CONNECTING MACHINE COMPONENTS TO TDSM}

In order to make the system easily extensible, TDSM provides two primary mechanisms for connecting new machine components to the system via implementation of a plugin interface:

1. The communication bus is a mechanism for efficiently managing streams of events within the system. The bus provides a medium for internal and external components to publish and subscribe to messages about activities in the system.

2. The breadboard is a mechanism for wiring machine components together. The breadboard enables explicit connections between machine components that allow for complex interactions among components.

3. Machine component plugins adapt machine behaviors to become available on the bus or breadboard for use within the TDSM ecosystem. Machine component plugins provide sets of interface definitions that allow internal or external components to participate in TDSM through software adapters.

Both the bus and the breadboard represent modular, flexible approaches to connecting various applications and external data sources to TDSM. Such extensibility supports transdisciplinarity by allowing team members from different disciplines to connect their preferred applications to the system and/or select from an existing ecosystem of applications that may help to improve outcomes. To illustrate this point, the following scenario describes one of many possible scenarios where the addition of a text analytics application to TDSM can assist in the generation of new and integrated knowledge.

\section{TDSM AND MACHINE ANALYTICS SCENARIO}

In this example, machine assistants help team members to understand how members from different disciplines use key concepts to communicate important information.

1. The team leader of a transdisciplinary research project activates a machine analytics application on TDSM.

2. Team members, representing a number of different disciplines, collaborate on training the machine to recognize key vocabulary (i.e., concept terms and jargon that are not already in the machine lexicon). 
3. Among a number of possible approaches, team members from each discipline compile representative collections of documents that are relevant to the project focus and feed each collection to the machine.

4. The machine uses various approaches to natural language processing to identify key terms from each collection and produce lists of the results by discipline.

5. Team members review the machine-generated lists and check off terms of interest.

6. The text analytics application adds the new vocabulary to its lexicon through a largely automated machine learning process.

7. The machine component is now capable of rapidly identifying key terms within large volumes of content (e.g., thousands of research articles in an online repository or Web-pages and files stored on servers around the world).

8. Given a source of online documents, the text analytics application engages in entity extraction. In brief, entity extraction involves locating and collecting entity references within targeted documents. This includes key terms from the training exercise and, optionally, other types of entities of interest such as people, places, and organizations.

9. Based on results of the entity extraction process, team members select a manageable subset of high-value documents for import to TDSM.

10. On import, TDSM converts machine analytics to Machine HyLights. When users open documents, key vocabulary and other entities of interest are highlighted and related metacontent (e.g., category, attributes, and associations), ferreted out by the machine, is displayed in the left session-margin.

11. With Machine HyLights stored in TDSM, users have the option to create mashups of all or selected Machine HyLights located within the collection of documents.

12. By capitalizing on the high-speed navigation capability of the mashup-margin, users can rapidly inspect Machine HyLights in their exact locations within their source documents.

13. As users navigate from Machine HyLight to Machine HyLight and from document to document, they evaluate the surrounding context of each highlighted entity. When users find Machine HyLights within fragments of text that express information of value, they add new HyLights that encompass the fragments and include their comments.

14. As a layer of machine and human HyLights accumulates across the collection of documents, members of each discipline create Knowledge Trails. Members arrange selected HyLights within the mashup margin in a preferred order, name and save the results to the server, and invite other team members to follow their travels through the collection.

15. Subsequently, users may export Knowledge Trails to the text analytics application as feedback to improve performance of the machine in assisting users in their sensemaking efforts. For example, Knowledge Trails provide data that may help the machine to recognize nonobvious associations between key concepts.

This scenario describes one of many possible use cases involving TDSM and currently available machine components. Given its modular and extensible design, TDSM may accommodate machine components of the future with potentially transformative capabilities for transdisciplinary research.

\section{THE CONVERSATION MODEL}

From a system architecture perspective, the framework for TDSM is a simple conversation (D. Boyer, personal communication, February 22, 2016). In this context, a conversation is any set of HyLights, created by humans or machines, with the following attributes: 
1. A conversation can have two types of users: people and machines.

2. A conversation has two types of roles: speakers with write permission and listeners with read only permission.

3. Users build conversations by (a) adding new HyLights to active conversations, (b) pulling in selected HyLights from existing conversations, and/or (c) combining existing conversations.

4. Users have the option to save conversations (e.g., Knowledge Trails) for reuse and sharing.

5. Conversations can be used to provide feedback to machines to improve their performance as sensemaking assistants.

6. In all instances, HyLights and conversations are preserved while related internal source documents and content remain immutable.

This combination of a simple model and indestructible history gives the system a Lego set quality with HyLights serving as reusable building blocks, conversations as pre-fabricated assemblies and sub-assemblies, and workflows as instructions for defined sensemaking activities and their outputs. This simple architecture establishes the foundation for a highly scalable and adaptable Social Machine that is especially compatible with the goals and values of transdisciplinarity.

\section{THREE EXAMPLES OF MACHINE-ASSISTED SENSEMAKING}

The concept of extended cognition, as first proposed by Clark and Chalmers (1998) and revised by others since (Bartlett, 2016), extends the periphery of mind beyond the boundary of the body. Mind is not merely embedded within but extends into the environment of the organism. From this perspective, mind encompasses artifacts that capture and represent knowledge shared by team members and technologies that support the development and use of the artifacts (Fiore \& Wiltshire, 2016). In the digital information age, extended cognition places intelligence into networks of interacting humans and machines (Ito, 2016). Arguably, this makes TDSM a type of external memory technology for representing the transactive memory system of a group through various boundary objects.

A transactive memory system is a mechanism for encoding, storing, and retrieving knowledge possessed by individual members of a team, including shared awareness of who holds what knowledge (Kotlarsky, den Hooff, \& Houtman, 2015). A boundary object is a tangible artifact that acts as a bridge for facilitating coordination and collaboration across disciplinary borders and maintaining shared representations of team knowledge. Boundary objects are a technology-based form of transactive memory that emerges from the transactive memory system of a team (Fiore \& Wiltshire, 2016).

TDSM provides three interacting mechanisms for producing various boundary objects to enhance sensemaking. These mechanisms, as described previously, include (a) a collaborative annotation system for pointing at high-value fragments, capturing related comments, and creating links between related annotations or HyLights, (b) a Knowledge Trail feature for producing preferred pathways through a document collection, and (c) a feedback loop that enables the coactive (i.e., on-going and close interaction on shared tasks; Bradshaw, 2013) emergence of human and machine intelligence. Among a number of potential benefits, TDSM may achieve its greatest impact by minimizing the time and effort required to navigate between related pieces of information located in the literature of different disciplines.

From the perspective of extended cognition (i.e., the idea that the physical apparatus of cognition encompasses both the individual organism and surrounding non-biological elements; Clark \& Chalmers, 1998; Smart, 2017), the main objectives of TDSM are to augment human sensemaking capabilities and make machines more capable of assisting humans in their sensemaking efforts. TDSM attempts to achieve these objectives by facilitating purposeful interactions and collaborations between humans and machines when tapping into multiple sources of online content (Scaife \& Rogers, 1996). To illustrate the potential of TDSM for supporting a transdisciplinary literature review, we 
describe how TDSM approaches each of the problems described previously (i.e., differences in language and meaning, information overload, and effects of cognitive bias).

\section{AN ELECTRONIC TRANSDISCIPLINARY CONCORDANCE}

To overcome differences between disciplines in such areas as epistemic dimensions, social dimensions, and knowledge production systems, effective collaboration requires the development of a shared language. TDSM addresses this problem through crowdsourcing of collaborative annotation activities to produce an electronic transdisciplinary concordance (ETC). A concordance is an alphabetical index of the principal words of a book with a reference to the passage in which each word occurs. Though similar to a standard concordance in some respects, ETC represents a new type of text in purpose, capabilities, production, and maintenance. Its main purpose is to assist members of transdisciplinary research teams in acquiring sufficient fluency in the terminology of multiple disciplines to participate effectively in the integration of ideas. In what follows, we briefly describe (a) how a reader may use ETC as a bridge across disciplinary literatures and (b) how ETC emerges from routine sensemaking activities enabled by TDSM.

\section{ETC in use}

While reading and marking up an article in TDSM, a user encounters an unfamiliar concept:

- The user selects the term within the article and opens ETC.

- Alternatively, the user opens ETC and enters the term in a search field or selects the term from an alphabetical index.

- ETC runs a search of accessible documents stored in TDSM (i.e., based on the security and permissions profile of the user) and displays the results as a Knowledge Trail.

- Each HyLight includes a tag indicating the disciplinary background of the author(s), a link to a full reference for the article, a link to the HyLight in its exact location within the source, and a list of terms that share the same or similar meaning.

Brown, Collins, and Duguid (1989) wrote that understanding the meaning of a concept means understanding its range of uses. ETC serves as a bridge across disciplinary literatures by situating selected terms within sets of exemplary HyLights to reveal multiple uses and shades of meaning.

\section{ETC as an emergent product}

ETC emerges as a product of the collective activity of TDSM users. Exemplary HyLights accumulate over time, as users annotate research literature across projects and departments at a single institution or on a larger, multi-institutional scale. While reading and marking up research articles in TDSM for a variety of purposes, a user will sometimes annotate a fragment that reveals the meaning of an important term through its context of use. The user who submitted the HyLight or anyone with privileges may add the HyLight to ETC:

- The user tags the HyLight with the targeted term and clicks a button to add exemplar status.

- The system automatically adds a discipline tag to the HyLight.

- TDSM has various administrative and analytic features for maintaining ETC as it grows.

In sum, ETC emerges through a crowdsourcing mechanism that captures the annotation activities of TDSM users. As long as TDSM is operating, users may continually add new terms and exemplars to the system. 


\section{DETECTION OF UNKNOWN UNKNOWNS}

Former Secretary of Defense, Donald Rumsfeld, speaking to the press in 2002, memorably used the phrase, "unknown unknowns," to refer to a problem that keeps analysts, researchers, and decision makers up at night:

There are known knowns; there are things we know that we know. There are known unknowns; that is to say there are things that, we now know we don't know. But there are also unknown unknowns - there are things we do not know we don't know (Defense Department Gov News, 2002)

In other words, a known unknown is a question that an analyst/researcher knows to ask and pursue further. An unknown unknown is a question that an analyst/researcher does not even know to ask. As an operational definition, within the context of TDSM, unknown unknowns are entities (e.g., key concept terms) in external sources that are strongly associated with entities of interest in a TDSM document collection or "conversation" but are not in the TDSM document collection and are unknown to participants in the conversation.

\section{A sufficiency criterion for transdisciplinary literature reviews}

TDSM helps transdisciplinary research teams determine the sufficiency of their literature reviews through an enhanced search mechanism:

- Team members create a Knowledge Trail of high-value HyLights from within their existing document collection.

- The project manager feeds the Knowledge Trail to the TDSM text analytics application.

- The machine finds all entities in the external repositories that are strongly related to highvalue entities in the document collection but do not appear in the collection (i.e., unknown unknowns, as operationally defined).

- The data that is returned to the researchers include (a) a list of entities in the external repository that have criterion-level relationships with entities in the TDSM collection and (b) a list of documents, ranked by likely value, that include the entities of interest.

- The researchers import files of interest to the TDSM collection and markup the new documents.

- The team repeats the process until satisfied with the sufficiency of their review.

TDSM makes high-speed text analytics compatible with deliberative, collaborative, human sensemaking practices. By adding a social component to machine analytics, TDSM establishes a feedback loop between machines and researchers that supports a systematic approach to the detection of unknown unknowns hidden in large volumes of content.

\section{MODERATING THE EFFECTS OF COGNITIVE BIASES}

Cognitive biases appear to be a side-effect of the survival imperative to make our cognitive machinery capable of rapidly filtering information. Arguably, the network effects of social media have amplified cognitive biases and made them increasingly pervasive in human thought and communications (Chamorro-Premuzic, 2014). In the context of transdisciplinary research projects, cognitive bias can be a major obstacle to achieving desired outcomes. As a general strategy for mitigating this tendency of mind, transdisciplinarity promotes the values of transparency, dialogue, self-reflection, and building trust among team members (Montuori, 2013). TDSM may contribute to implementing this strategy by enabling interactions between humans and machines that support these core values. To illustrate this potential, we describe a number of TDSM capabilities that add transparency and opportunities for dialogue during collaborative sensemaking activities. 


\section{Every HyLight has its own address}

As users markup documents in performing a literature review, TDSM assigns a unique URL to each user-generated HyLight. This endows HyLights with a number of key affordances that may contribute to transparency and related values:

- Users can create links between HyLights in different documents and describe the associations. Identifying non-obvious associations, especially between distantly related sources, creates opportunities for serendipity and the generation of new ideas. This record of links between HyLights located in different documents is available to team members for review and dialogue.

- When HyLights move to external locations (e.g., to Word documents, GoogleDocs, visualizations, and spreadsheets), each HyLight has a link or tether back to its exact location in its source. Accessing a HyLight in its source provides evidence for assessing the quality or provenance of the information. This includes information related to lineage-what is the origin of this information, and pedigree-how reliable is the source of this information.

\section{Mapping the intellectual travels of a team through a document collection}

TDSM enables teams to have discussions tied to fragments of text selected across collections of documents in a controlled, organized, efficient, and auditable manner. The system maintains an historical record for auditing past decisions, informing future actions, and as a source of analytics for assessing performance. TDSM manages accumulating and overlapping markup through various mechanisms:

- A color-coding feature for mapping the intellectual travels of team members through a document collection.

- The capacity to align commentary in the margin with related fragments in a document.

- The capacity to quickly navigate between related HyLights within and between documents (e.g., HyLights located in the literature of different disciplines).

- The capacity to organize and display HyLights in Knowledge Trails, graphic visualizations, and other representations.

In sum, TDSM maps the thinking of readers across document collections, enables readers to share their HyLights, and provides a variety of options for displaying and navigating the terrain. Through these and related mechanisms, TDSM supports rich feedback, self-reflection, transparency, and other key enabling values for transdisciplinary research.

\section{CONCLUSION}

Transdisciplinary research teams face formidable challenges in adopting a holistic perspective on the nature of knowledge and knowing. Social Machines, as an approach to unifying machines and social processes, are a promising approach to enhancing transdisciplinarity as a research strategy. We have described how a Transdisciplinary Social Machine (TDSM), tuned to the requirements of a literature review, may assist team members in overcoming differences in language and meaning, information overload, and effects of cognitive bias.

TDSM helps non-technical users (and technical users, too) to find, organize, and synthesize highvalue information from multiple sources. Among other desirable effects, it assists team members in creating new knowledge of value and maintains an historical record of conversations tied to important sections of documents for auditing past decisions and informing future actions. TDSM provides these benefits by assimilating machine analytics and other machine components into collaborative sensemaking networks. This approach redefines the human/machine division of labor to make machine-scale analytics more compatible with human-scale sensemaking practices. As a consequence of interacting with the system, users become more capable of thinking within a domain or problem 
space and machines become more capable of assisting users in their sensemaking efforts. In sum, the system establishes a feedback loop for the continuous improvement of human and machine intelligence.

In addition to supporting transdisciplinarity, TDSM may help to prepare students for the $21^{\text {st }}$ century information economy and an uncertain future. As rapid technological change eliminates jobs in every sector and new types of work are emerging that require new and different skill sets, the curricula in schools remain largely out of sync with global economic reality (Soule \& Warrick, 2015). As an historical analogy, literacy in Western Europe until the fifteenth century was largely limited to wealthy elite, clergy, and scholars. With the arrival of Gutenberg's innovation and the availability of low-cost reading material, literacy spread widely, especially when newspapers appeared in the 1600s. By analogy, the Internet, smart machines, and related technologies are the movable type and printing press of today, and data scientists are the elite literate. Similarly, as the spread of literacy helped to fuel the growth of a merchant class and usher in the Renaissance period, the spread of Social Machines for collaborative sensemaking will help fuel a resurgence of the diminished middle-class and, potentially, contribute to a global transformation of society (Lanier, 2013).

\section{REFERENCES}

Babcock, L., Loewenstein, L., Issacharoff, S., \& Camerer C. (1995). Biased judgments of fairness in bargaining. American Economic Review, 85, 128-141.

Bartlett, G. (2016). Extended cognition and the extended mind: Introduction. Essays in Philosophy, 17(2), 1-7. https://doi.org/10.7710/1526-0569.1554

Berners-Lee, T. and Fischetti, M. (1999). Weaving the Web: the original design and ultimate destiny of the World Wide Web by its inventor / Tim Berners-Lee with Mark Fischetti. San Francisco: Harper.

Boon, S. (2016, January 7). 21st century science overload [Blog post]. Retrieved from http://www.cdnsciencepub.com/blog/21st-century-science-overload.aspx

Bradshaw J. M. (2013). From knowledge science to symbiosis science. International Journal of Human Computer Studies, 71(2), 171-176. https://doi.org/10.1016/j.ijhcs.2012.10.003

Brown, J. S., Collins, A., \& Duguid, P. (1989). Situated cognition and the culture of learning. Educational Researcher, 18(1), 32-42. https://doi.org/10.3102/0013189X018001032

Buregio, V., Meira, S. \& Rosa, N. (2013). Social machines: A unified paradigm to describe social web-oriented systems. WWW '13 Companion Proceedings of the 22nd International Conference on World Wide Web (pp. 885-890). https://doi.org/10.1145/2487788.2488074

Canas, J. J., Fajardo, I., \& Salmeron, L. (2006). Cognitive flexibility. In W. Karwowski (Ed.), International encyclopedia of ergonomics and buman factors (pp. 297-300).

Chamorro-Premuzic, T. (2014, May 13). How the web distorts reality and impairs our judgement skills. Retrieved from https://www.theguardian.com/media-network/media-network-blog/2014/may/13/internetconfirmation-bias

Choi, B. K., \& Pak, A. P. (2006). Multidisciplinarity, interdisciplinarity, and transdisciplinarity in health research, services, education and policy: 1 . Definitions, objectives, and evidence of effectiveness. Clinical and Investigative Medicine, 29, 351-364.

Chugh, D., \& Bazerman, M. H. (2007). Bounded awareness: What you fail to see can hurt you. Mind \& Society, 6, 1-18. https://doi.org/10.1007/s11299-006-0020-4

Cilliers, P. (1998). Complexity and postmodernism. Understanding complex systems. London: Routledge.

Clark, A., \& Chalmers, D. (1998). The extended mind. Analysis, 58(1), 7-19. https://doi.org/10.1093/analys/58.1.7

Darbellay, F. (2014). Rethinking inter- and transdisciplinarity: Undisciplined knowledge and the emergence of a new thought style. Futures, 65, 163-174. https://doi.org/10.1016/i.futures.2014.10.009 
Defense Dept Government News Transcript: DoD News Briefing. (2002, Feb. 12). Secretary Rumsfeld and General Myers, United States Department of Defense. Retrieved from http://archive.defense.gov/Transcripts/Transcript.aspx?TranscriptID $=2636$

Fay, R., \& Montague, N. R. (2015). Witnessing your own cognitive bias: A compendium of classroom exercises. Issues in Accounting Education, 30(1), 12-26. https://doi.org/10.2308/iace-50919

Fiore, S. M., \& Wiltshire, T. J. (2016). Technology as teammate: Examining the role of external cognition in support of team cognitive processes. Frontiers in Psychology, 7(1531), 1-17. https://doi.org/10.3389/fpsyg.2016.01531

Golman, R., Hagmann, D., \& Loewenstein G. (2017). Information avoidance. Journal of Economic Literature, 55(1), 96-135. https://doi.org/10.1257/jel.20151245

Greene, J., Azevedo, R., \& Torney-Purta, J. (2008). Modeling epistemic and ontological cognition: Philosophical perspectives and methodological directions. Educational Psychologist, 43, 142-160. https://doi.org/10.1080/00461520802178458

Hall K. L., Stokols D., Moser R., Taylor B., Thornquist M., Nebeling L., \& Jeffery R. W. (2008). The collaborative readiness of transdisciplinary research teams and centers: Findings from the National Cancer Institute's TREC year-one evaluation study. American Journal of Preventive Medicine, 35(2), 161-172. https://doi.org/10.1016/j.amepre.2008.03.035

Hall, K. L., \& Vogel, A. L. (2012). A four-phase model of translational team-based research: Goals, team processes, and strategies. Translational Behavioral Medicine, 2, 415-430. https://doi.org/10.1007/s13142-012$\underline{0167-\mathrm{y}}$

Hofer, B. (2004). Exploring the dimensions of personal epistemology in differing classroom contexts: Student interpretations during the first year of college. Contemporary Educational Psychology, 29, 129-163. https://doi.org/10.1016/j.cedpsych.2004.01.002

Kahneman, D., Slovic, P., \& Tversky, A. (1982). Judgment under uncertainty: Heuristics and biases. New York, NY: Cambridge University Press. https://doi.org/10.1017/CBO9780511809477

Klein, G., Moon, B., \& Hoffman, R. R. (2006). Making sense of sensemaking 1: Alternative perspectives. IEEE Intelligent Systems, 21, 70-73. https://doi.org/10.1109/MIS.2006.75

Klein, G., Phillips, J. K., Rall, E. L., \& Peluso, D. (2007). A data frame theory of sensemaking. In R. A. Hoffman (Ed.), Expertise out of context: Proceedings of the Sixth International Conference on Naturalistic Decision Making, (pp. 113-153). New York, NY: Taylor \& Francis Group.

Klein, J. T. (2008a). The rhetoric of interdisciplinarity. In A. Lunsford, K. Wilson \& R. Ebeerly (Eds.), The Sage handbook of rhetorical studies (pp. 265-284). Thousand Oaks, CA: Sage

Klein, J. T. (2008b). Evaluation of interdisciplinary and transdisciplinary research: Aliterature review. American Journal of Preventive Medicine, 35, 116-123. https://doi.org/10.1016/j.amepre.2008.05.010

Knorr-Cetina, K. (1999). Epistemic cultures: How science makes knowledge. Cambridge, MA: Harvard University Press.

Kotlarsky, J., den Hooff, B., \& Houtman, L. (2015). Are we on the same page? Knowledge boundaries and transactive memory system development in cross-functional teams. Communication Research 42(3), 319-344. https://doi.org/10.1177/0093650212469402

Ito, J. (2016, February 10). Extended intelligence. Retrieved from https://pubpub.ito.com/pub/extendedintelligence

Lavallée, M., Robillard, P. N., \& Mirsalari, R. (2014). Performing systematic literature reviews with novices: An iterative approach. IEEE Transactions on Education, 57(3), 175-181.

https://doi.org/10.1109/TE.2013.2292570

Lanier, J. (2013). Who owns the future? New York, NY: Simon and Schuster.

Mathieu, J. E., Maynard, M. T., Rapp, T., \& Gilson, L. (2008). Team effectiveness 1997-2007: A review of recent advancements and a glimpse into the future. Journal of Management, 34, 410-476.

https://doi.org/10.1177/0149206308316061 
A Social Machine for Transdisciplinary Research

Misra, S., Hall, K., Feng, A., Stipleman, B., \& Stokols, D. (2011). Collaborative processes in transdisciplinary research. In M. Kirst, N. Schaefer-McDaniel, S. Hwang, \& P. O' Campo (Eds.), Converging disciplines: A transdisciplinary research approach to urban health problems (pp. 97-110). New York: Springer.

Misra, S., Stokols, D., \& Cheng, L. (2015). The transdisciplinary orientation scale: Factor structure and relation to the integrative quality and scope of scientific publications. Journal of Translational Medicine and Epidemiology, 3(2), 1042.

Montuori, A. (2013). The complexity of transdisciplinary literature reviews. Complicity: An International Journal of Complexity and Education, 10(1-2), 45-55.

Mørk, B. E., Aanestad, M., Grisot, M., \& Hanseth, O. (2008). Conflicting epistemic cultures and obstacles for learning across communities of practice. Knowledge and Process Management, 15(1), 12-23.

https://doi.org/10.1002/kpm.295

Mullane, K., \& Williams, M. (2013). Bias in research: The rule rather than the exception? Elsevier Editors' Update, 40, 7-10. Retrieved from https://www.elsevier.com/editors-update/story/publishing-ethics/bias-inresearch-the-rule-rather-than-the-exception

Pirolli, P., \& Card, S. (2005). The sensemaking process and leverage points for analyst technology as identified through cognitive task analysis. Proceedings of International Conference on Intelligence Analysis, 2-4.

Pohl, C. (2010). From transdisciplinarity to transdisciplinary research. Transdisciplinary Journal of Engineering \& Science, 1(1), 74-83.

Rabin, M., \& Schrag. J. L. (1999). First impressions matter: A model of confirmatory bias. Quarterly Journal of Economics, 114(1), 37-82. https://doi.org/10.1162/003355399555945

Richter, T. (2011). Cognitive flexibility and epistemic validation in learning from multiple texts. In J. Elen, E. Stahl, R. Bromme, \& G. Clarebout (Eds.), Links between beliefs and cognitive flexibility (pp. 125-40). Berlin: Springer. https://doi.org/10.1007/978-94-007-1793-0 7

Richter, T., \& Maier, J. (2017). Comprehension of multiple documents with conflicting information: A two-step model of validation. Educational Psychologist, 52(3), 148-166. https://doi.org/10.1080/00461520.2017.1322968

Robinson, J. (2008). Being undisciplined: Transgressions and intersections in academia and beyond. Futures, 40, 70-86. https://doi.org/10.1016/i.futures.2007.06.007

Rosenfield, P. L. (1992). The potential of transdisciplinary research for sustaining and extending linkages between the health and social sciences. Social Science \& Medicine, 35, 1343-1357. https://doi.org/10.1016/0277-9536(92)90038-R

Rumelhart, D. E., \& Norman, D. A. (1978). Accretion, tuning and restructuring: Three modes of learning. In J. W. Cotton \& R. L. Klatzky (Eds.), Semantic factors in cognition (pp. 37-53). Hillsdale NJ: Erlbaum.

Salazar M. R., Lant T. K., Fiore S. M., \& Salas E. (2012). Facilitating innovation in diverse science teams through integrative capacity. Small Group Research, 43, 527-558. https://doi.org/10.1177/1046496412453622

Scaife, M., \& Rogers, Y. (1996). External cognition: How do graphical representations work? International Journal of Human-Computer Studies, 45, 185-213. https://doi.org/10.1006/ijhc.1996.0048

Schacter, D. L. (2001). The seven sins of memory. New York, NY: Houghton Mifflin.

Schraw, G. J., Bendixen, L. D., \& Dunkle, M. E. (2002). Development and validation of the Epistemic Belief Inventory (EBI). In B. K. Hofer \& P. R. Pintrich (Eds.), Personal epistemology: The psychology of beliefs about knowledge and knowing (pp. 261-275). Mahwah, NJ: Lawrence Erlbaum Associate.

Shanahan, T., \& Shanahan, C. (2008). Teaching disciplinary literacy to adolescents: Rethinking content-area literacy. Harvard Educational Review, 78(1), 40-59. https://doi.org/10.17763/haer.78.1.v62444321 p602101

Shattuck, L. G., \& Miller, N. L. (2006). Extending naturalistic decision making to complex organizations: A dynamic model of situated cognition. Organization Studies, 27, 989-1009.

https://doi.org/10.1177/0170840606065706 
Shrivastava, P., \& Ivanaj, S. (2011). Transdisciplinary art, technology, and management for sustainable enterprise. Transdisciplinary Journal of Engineering and Science, 2, 81-92.

Slaney, M., \& Russell, D. M. (2005). Measuring information understanding in large document collections. Proceedings of the 38th Annual Hawaii International Conference on System Sciences, 105-114. https://doi.org/10.1109/HICSS.2005.404

Smart, P. R. (2017). Situating machine intelligence within the cognitive ecology of the internet. Minds \& Machines, 27, 357-380. https://doi.org/10.1007/s11023-016-9416-Z

Smart, P. R., \& Shadbolt, N. R. (2014). Social machines. In M. Khosrow-Pour (Ed.) Encyclopedia of information science and technology (pp. 6855-6862). Hershey, PA: IGI Global.

Smart, P. R., Simperl, E., \& Shadbolt, N. (2014). A taxonomic framework for social machines. In D. Miorandi, V. Maltese, M. Rovatsos, A. Nijholt, \& J. Stewart (Eds.), Social collective intelligence: Combining the powers of bumans and machines to build a smarter society (pp. 51-85). Berlin: Springer. https://doi.org/10.1007/978-3-319$\underline{08681-1 \quad 3}$

Smith, J., \& Noble H. (2014). Bias in research. Evidence-Based Nursing, 17, 2-3. https://doi.org/10.1136/eb2014-101946

Soulé, H. \& Warrick, T. (2015. Defining 21st century readiness for all students: What we know and how to get there. Psychology of Aesthetics, Creativity, and the Arts, 9(2), 178-186. https://doi.org/10.1037/aca0000017

Stock, P. \& Burton R. J. (2011). Defining terms for integrated (multi-inter-trans-disciplinary) sustainability research. Sustainability, 3(8), 1090-1113. https://doi.org/10.3390/su3081090

Stokols D. (2014). Training the next generation of transdisciplinarians. In M. O'Rourke, S. Crowley, S. D. Eigenbrode, \& J. D. Wulfhorst (Eds.), Enhancing communication \& collaboration in interdisciplinary research (pp. 56-81). Los Angeles, CA: Sage Publications. https://doi.org/10.4135/9781483352947.n4

Vilsmaier, U., Brandner, V., \& Engbers, M. (2017). Research in-between: The constitutive role of cultural differences in transdisciplinarity. In H. Dieleman, B. Nicolescu, \& A. Ertas (Eds.), Transdisciplinary \& interdisciplinary education and research (pp. 89-102). USA: Atlas Publishing.

Walsh, J. P., \& Fahey, L. (1986). The role of negotiated belief structures in strategy making. Journal of Management 12(3), 325-338. https://doi.org/10.1177/014920638601200302

Weick, K., Sutcliffe, K. M., \& Obstfeld, D. (2005), Organizing and the process of sensemaking. Organization Science, 16, 409-421.

\section{BIOGRAPHY}

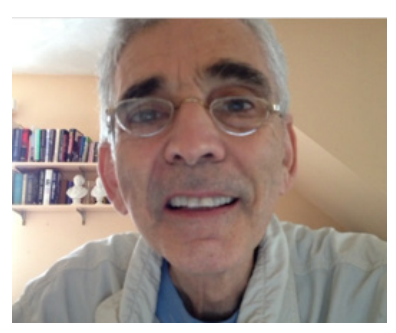

David Lebow is the inventor of HyLighter and CEO of HyLighter LLC. David received his Ph.D. in Instructional Systems Design from Florida State University in 1995 and has won several national awards for his work in the area of computer-enhanced collaborative learning environments. 